IOS Press

\title{
Editorial
}

\section{Advancing berry research in cancer}

\author{
Pan Pan* and Li-Shu Wang* \\ Department of Medicine, Division of Hematology and Oncology, Medical College of Wisconsin, USA
}

Cancer is the second leading cause of death worldwide, having caused 8.8 million deaths in 2015 . That is nearly 1 in 6 of all global deaths. Around one third of deaths from cancer are due to the 5 leading behavioral and dietary risks: high body mass index, low fruit and vegetable intake, lack of physical activity, tobacco use, and alcohol use. Berries include many commonly consumed fruits and vegetables, such as strawberries, blueberries, blackberries, red raspberries, black raspberries, cranberries, grape, kiwi, banana, tomatoes, eggplant, cucumber, watermelon, etc., as well as many uncommon types, such as gooseberries, Goji berries, elderberries, noni (Morinda citrifolia), and acai (Euterpe oleracea Mart), etc. Berries are known to contain abundant phytochemicals and natural bioactive compounds, which have been reported as anti-cancer agents. Therefore, the beneficial effects and the underlying mechanisms of berries against cancers are important topics in cancer research. Articles in this special issue reported the recent advances of berry research in cancers including human efficacy trials and the use of animal models and cell culture systems to elucidate mechanisms of action. The topics include, but are not limited to, strategies to enhance bioavailability of berry phytochemicals for cancer therapeutics, the role of gut bacteria in berry-mediated cancer prevention, anti-inflammation as the major anti-cancer mechanism using berries, berries' effects on H. pylori and gastric cancer, etc. Overall, the findings not only support the use of berries to reduce cancer risk but also explore the potential use of berry phytochemicals for cancer therapeutics.

\footnotetext{
*Corresponding authors: Pan Pan and Li-Shu Wang, Department of Medicine, Division of Hematology and Oncology, Medical College of Wisconsin, USA. E-mail: liswang@mcw.edu.
} 